\title{
Interference Alignment for the $K$-user MIMO X Network Using Time Division Multiple Access
}

\author{
Sudheesh P. G.*, Maurizio Magarini ${ }^{\dagger}$, P. Muthuchidambaranathan* \\ *Department of Electronics and Communication Engineering \\ National Institute of Technology, 620015 Tiruchirappalli, India \\ Email:pgsudheesh@gmail.com,muthuc@nitt.edu \\ $\dagger^{\dagger}$ Dipartimento di Elettronica, Informazione e Bioingegneria \\ Politecnico di Milano, 20133 Milano, Italy \\ Email: maurizio.magarini@polimi.it
}

\begin{abstract}
This paper investigates an interference alignment (IA) scheme suitable for a $K$-user multiple-input multipleoutput (MIMO) $\mathbf{X}$ network. The $K$-user MIMO $X$-network is a communication architecture where each transmitter, equipped with multiple antennas, has independent messages for each of the receivers, also equipped with multiple antennas. Earlier only $2 \times N$ or $M \times 2 \mathbf{X}$ networks was considered to be achievable. In this paper we remove this restriction by employing time division multiple access scheme. The proposed IA scheme allows to achieve a sum degree of freedom of $\frac{K^{2} A}{2 K-1}$, where $A$ is the number of antennas at each transmitter and receiver. The capacity and bit error rate performance of the proposed scheme is compared to that of another scheme recently proposed by Park and Ko. Simulation results are reported to show the bit error-rate performance for $K$-user (for a toy example, $K$ is set as 3) and two-user $X$ channel IA in case of transmission over flat-fading Rayleigh channels.
\end{abstract}

\section{INTRODUCTION}

The capacity of wireless networks provides important information on the usability of wireless networks for different applications. In a generelized form, the capacity of a network versus SNR can be approximated as

$$
C(\mathrm{SNR})=d \cdot \log (\mathrm{SNR})+\mathcal{O}(\log (\mathrm{SNR}))
$$

where $d$ defines the number of degrees of freedom (DoF) and SNR is the signal-to-noise ratio of the network. Recently DoF of $K$-user X networks [1] and interference networks [2] have been characterized.

In the context of distributed multiple-input multiple output (MIMO) wireless networks, an interference channel reveals the communication scenario of transmitter-receiver pairs in which each transmitter sends information for its intended receiver and, at the same time, creates interference to the other receivers [1]-[5]. The X network adds another feature to the interference channel in the sense that not only each transmitter has a message for its corresponding receiver, but it also has other independent messages for each of the other receivers [1], [3], [4].

Different approaches can be adopted to deal with interference which arises in wireless networks [2], [5]-[7]. Among these, interference alignment (IA) is a technique where interfering signals at each receiving node are confined into a subspace which does not contain that spanned by the signal of interest. The desired signal can therefore be recovered free from interference [2], [3], [7]. The fundamental concepts related to IA were introduced in [2], with emphasis on the temporal domain where a joint design of pre-coding matrices over multiple symbol extensions of the time-varying channels is proposed. Although [2] focuses on the temporal dimension, extension to other dimensions, i.e. frequency, space and codes, is straightforward.

A crucial assumption in [2] is that channel state information (CSI) is globally available at each transmitting node. Global CSI knowledge allows us to write closed form expressions for the pre-coding matrices. However, even if global knowledge is available, closed form expressions for the pre-coding matrices can be written only when the number of users is less than three [2]. Perfect IA for X network is applicable with either two transmitter or with two receiver [4]. This is broadly defined as $2 \times N$ or $M \times 2 \mathrm{X}$ networks.

Partial IA for $M \times N$ networks has been proposed in [4] where it also shown that the achievable degree of freedom (DoF) is between the lowerbound $\frac{M N A}{M+N-1 / A}$ and the upperbound $\frac{M N A}{M+N-1}$, being $A$ the number of antennas that are available at both the ends of each link. However, the perfect IA of $M \times N \mathrm{X}$ networks is still an open research topic. In a recent paper [12] Park and Ko propose an approach for IA that allows to achieve perfect alignment without time extension at the cost of a slightly lesser DoF. For the $K$-user X network, the scheme proposed by Park and Ko achieves a sum-DoF of $K A / 2$, where $A=2 K$.

In this paper, we propose a scheme where perfect IA is achieved for a $K$-user MIMO X network with $A=2 K-1$. For the proposed scheme, we assume that CSI between all the links is known both at the transmitters and receivers. For example, for receiver 1 , CSI is required for designing precoding and zeroforcing vectors based on (11),(12),(13),(14) and (15). Moreover, a block Rayleigh fading assumption is done where the coefficients of MIMO channels remain constant for the entire duration of the transmission. In the proposed scheme IA is achieved at each receiver using time division multiple access (TDMA). Error rate performance is evaluated for already proposed two-user IA and $K$-user IA, where $K$ is taken as 3 for a fair comparison.

The paper is organized as follows. A background on IA is 
provided in Section II. In Section III the proposed approach for $K$-user MIMO X network is demonstrated considering the three-user case as an example. Section IV explains numerical and simulation results. Conclusions are drawn in Sec. V.

\section{Notation}

The following notation is used in the paper: $(.)^{T}$ correspond to transpose. (. $)^{-1}$ is used for inverse of matrix. Vectors and matrices are represented by lower case and upper case boldface letters, respectively.

\section{II. $K$-USER MIMO X NETWORK IA}

For a $K$-user MIMO X network where each transmitting and receiving node has $A$ antennas, the $A \times 1$ received signal vector for the $j$-th user is

$$
\mathbf{y}_{i}=\sum_{i=1}^{K} \mathbf{H}_{i j} \mathbf{x}_{j}+\mathbf{n}_{i}, \quad i=1, \ldots, K,
$$

where $\mathbf{x}_{j}$ is the $A \times 1$ signal vector transmitted by the $j$-th user, $\mathbf{H}_{i j}$ is a $A \times A$ channel matrix between transmitter $j$ and receiver $i$, with $i, j \in\{1,2, \ldots, K\}$, whose elements are assumed to be independent and identically distributed (i.i.d) complex Gaussian random variables with zero mean and unit variance, and $\mathbf{n}_{i}$ is a $A \times 1$ vector of i.i.d. complex Gaussian random variables with zero mean and variance $\sigma_{n}^{2}$. Each transmitter is intended to transmit independent messages to each of the receivers separately. The signal vector transmitted by the $j$-th user is

$$
\mathbf{x}_{j}=\sum_{i=1}^{K} \mathbf{b}_{i j} x_{i j}
$$

where $x_{i j}$ is the message to be transmitted from transmitter $j$ to receiver $i$ and $\mathbf{b}_{i j}$ is the beamforming vector associated with $x_{i j}$.

In the following we will consider the $K$-user case. By substituting (3) in (2) we get

$$
\mathbf{y}_{i}=\sum_{j=1}^{K} \mathbf{H}_{i j} \sum_{l=1}^{K} \mathbf{b}_{l j} x_{l j}+\mathbf{n}_{i}, \quad i=1, \ldots, K .
$$

The above equation can be rewritten as

$$
\mathbf{y}_{i}=\sum_{j=1}^{K} \mathbf{H}_{i j} \mathbf{b}_{i j} x_{i j}+\sum_{j=1}^{K} \sum_{l=1, l \neq i}^{K} \mathbf{H}_{i j} \mathbf{b}_{l j} x_{l j}+\mathbf{n}_{i},
$$

where the first and second term at the RHS are the signal vector intended for the $i$-th receiver and the interference affecting it, respectively.

In order to satisfy the IA condition interfering signals in each receiver must span the same subspace. For aligning interference at the receiver 1 the following conditions must be satisfied

$$
\left.\begin{array}{c}
\operatorname{SPAN}\left\{\mathbf{H}_{11} \mathbf{b}_{21}\right\}=\operatorname{SPAN}\left\{\mathbf{H}_{12} \mathbf{b}_{22}\right\}=\cdots=\operatorname{SPAN}\left\{\mathbf{H}_{1 K} \mathbf{b}_{2 K}\right\} \\
\vdots \\
\operatorname{SPAN}\left\{\mathbf{H}_{11} \mathbf{b}_{K 1}\right\}=\operatorname{SPAN}\left\{\mathbf{H}_{12} \mathbf{b}_{K 2}\right\}=\cdots=\operatorname{SPAN}\left\{\mathbf{H}_{1 K} \mathbf{b}_{K K}\right\}
\end{array}\right\}
$$

where eq. (6) aligns the interference corresponding to signals intended for receiver 2 and the interference corresponding to signals intended for receiver $K$. Similarly, for aligning interference at receivers 2 till $K$, the following conditions must be satisfied

$$
\left.\begin{array}{c}
\operatorname{SPAN}\left\{\mathbf{H}_{21} \mathbf{b}_{11}\right\}=\operatorname{SPAN}\left\{\mathbf{H}_{22} \mathbf{b}_{12}\right\}=\cdots=\operatorname{SPAN}\left\{\mathbf{H}_{2 K} \mathbf{b}_{1 K}\right\} \\
\vdots \\
\operatorname{SPAN}\left\{\mathbf{H}_{21} \mathbf{b}_{K 1}\right\}=\operatorname{SPAN}\left\{\mathbf{H}_{22} \mathbf{b}_{K 2}\right\}=\cdots=\operatorname{SPAN}\left\{\mathbf{H}_{2 K} \mathbf{b}_{K K}\right\}
\end{array}\right\}
$$

$$
\begin{gathered}
\operatorname{SPAN}\left\{\mathbf{H}_{K 1} \mathbf{b}_{11}\right\}=\operatorname{SPAN}\left\{\mathbf{H}_{K 2} \mathbf{b}_{12}\right\}=\cdots=\operatorname{SPAN}\left\{\mathbf{H}_{K K} \mathbf{b}_{1 K}\right\} \\
\vdots \\
\operatorname{SPAN}\left\{\mathbf{H}_{K 1} \mathbf{b}_{(K-1) 1}\right\}=\cdots=\operatorname{SPAN}\left\{\mathbf{H}_{K K} \mathbf{b}_{(K-1) K}\right\}
\end{gathered}
$$

For aligning interference vectors in receiver 1 , a possible choice of beamforming vectors is obtained from (6).

$$
\begin{gathered}
\mathbf{b}_{22}=\mathbf{H}_{12}^{-1} \mathbf{H}_{11} \mathbf{b}_{21} \\
\vdots \\
\mathbf{b}_{2 K}=\mathbf{H}_{1 K}^{-1} \mathbf{H}_{11} \mathbf{b}_{21} .
\end{gathered}
$$

For receiver 2, interference due to symbols intended for receiver 1 are aligned along a common direction as given in (7). Similarly symbols intended for receiver 3 are aligned along another independent direction. The same strategy is extended till receiver $K$ as in (8), that is we fix a vector direction for each receivers' intended symbol. Together with the alignment conditions, it is also required that desired signals vectors can be chosen to be linear independent of the interference dimensions at each receiver. As shown in [4] the construction of beamforming vectors that simultaneously satisfy all the conditions is not feasible. As a consequence the schemes to achieve perfect IA for the $M=2$ and $N=2$ described in [4], cannot be extended to the $K$-user case.

\section{Proposed PERFECT $K$-USER INTERFERENCE ALIGNMENT SCHEME}

Consider the singular value decomposition (SVD) of the channel matrix $\mathbf{H}=\mathbf{U} \boldsymbol{\Sigma} \mathbf{V}^{H}$, where $\boldsymbol{\Sigma}$ is a diagonal matrix of singular values and $\mathbf{U}, \mathbf{V}$ are two unitary matrices containing left and right singular vectors of $\mathbf{H}$, respectively. The algorithm here proposed implements IA using the knowledge of the eigen structure of channel matrices.

\section{A. Designing Precoder for Proposed IA Scheme}

From the knowledge of eigen structure, one of the interfering signals is chosen to define the reference direction in each receiver separately according to a TDMA scheme. For example, in order to align interference at receiver 1 we use time slot 1 . Interference is aligned in receiver 1 choosing $\mathbf{H}_{11} \mathbf{b}_{21}$ to $\mathbf{H}_{11} \mathbf{b}_{K 1}$ as the $K-1$ reference directions. Along these directions interference corresponding to desired signals of receiver 2 to receiver $K$ will be aligned.

The block diagram of the considered $K$-user MIMO $\mathrm{X}$ network is shown in Figure 1. To align interference at 


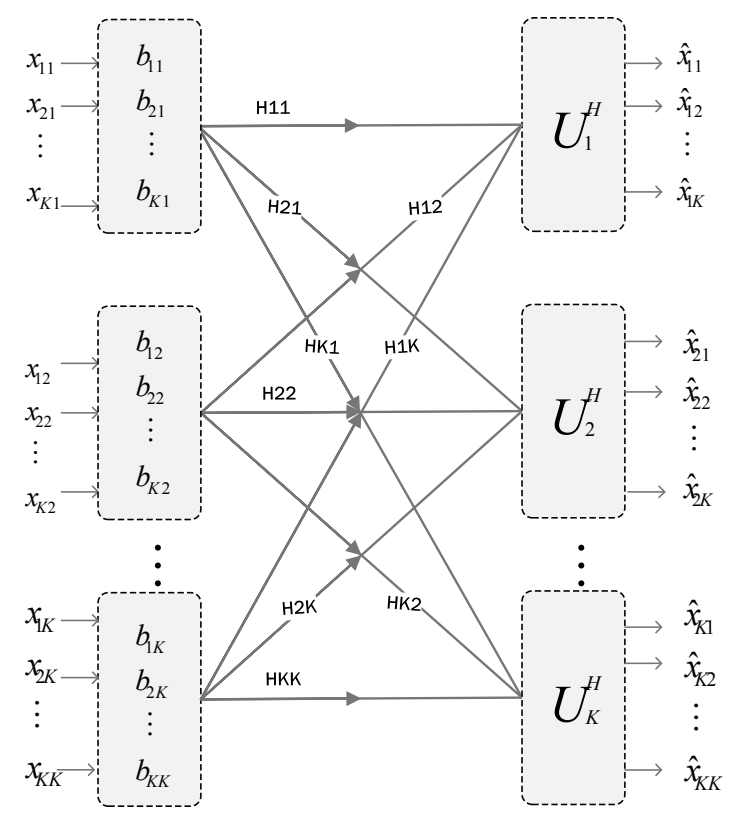

Fig. 1. Interference alignment on the $K$-user MIMO X network.

receiver 1 due to the desired signals of receiver 2 , we choose beamforming vectors $\mathbf{b}_{22}$ to $\mathbf{b}_{2 K}$ as follows.

$$
\begin{gathered}
\mathbf{b}_{22}=\mathbf{H}_{12}^{-1} \mathbf{H}_{11} \mathbf{b}_{21} \\
\vdots \\
\mathbf{b}_{2 K}=\mathbf{H}_{1 K}^{-1} \mathbf{H}_{11} \mathbf{b}_{21}
\end{gathered}
$$

Similarly, to align interference due to the desired signals of receiver $K$, we choose

$$
\begin{gathered}
\mathbf{b}_{K 2}=\mathbf{H}_{12}^{-1} \mathbf{H}_{11} \mathbf{b}_{K 1} \\
\vdots \\
\mathbf{b}_{K K}=\mathbf{H}_{1 K}^{-1} \mathbf{H}_{11} \mathbf{b}_{K 1},
\end{gathered}
$$

where beamforming vectors $\mathbf{b}_{11}, \mathbf{b}_{21}$, till $\mathbf{b}_{K 1}$ are chosen as the $K$ right singular vectors associated with the $K$ lowest singular values of $\mathbf{H}_{11}$. To retrieve the desired signal of receiver 1 from transmitter $2\left(x_{21}\right)$, beamforming vector $\mathbf{b}_{12}$ is chosen as the right singular vector associated with the minimum singular value of $\mathbf{H}_{12}$. Similarly $\mathbf{b}_{1 K}$ is chosen as the right singular vector associated to the minimum singular value of $\mathbf{H}_{1 K}$. This allows the desired signal to be retrieved by zero forcing algorithm and undesired signal to align along $\mathbf{H}_{11} \mathbf{b}_{21}$ to $\mathbf{H}_{11} \mathbf{b}_{K 1}$. In other words, out of $2 K-1$ dimensions, desired signal occupies $K$ dimensions and interference gets aligned to rest of the dimensions, which makes DoF of $\frac{K^{2}}{2 K-1}$ for $K$-user X network.

Aligning interference at receiver 2 in time slot 2 requires another set of beamforming vectors at the transmitters. This is because $\mathbf{b}_{21}$ to $\mathbf{b}_{K 1}$ are found from (6) and used for aligning interference at receiver 1 . In order to perform interference alignment at receiver 2 , we need to find another set of vectors which satisfies (7). Beamforming vectors $\mathbf{b}_{11}, \mathbf{b}_{21}$ to $\mathbf{b}_{K 1}$ are chosen as the right singular vectors associated with the $K$ minimum singular values of $\mathbf{H}_{11} \cdot \mathbf{b}_{22}$ is taken as a singular vector of $\mathbf{H}_{22}$ and $\mathbf{b}_{2 K}$ is taken as an singular vector of $\mathbf{H}_{2 K}$. Similarly, aligning interference at receiver $K$ requires another set of beamforming vectors at the transmitters. Beamforming vectors $\mathbf{b}_{11}, \mathbf{b}_{21}$ and $\mathbf{b}_{K 1}$ are the singular vectors of $\mathbf{H}_{11}$. The vector $\mathbf{b}_{K 2}$ is taken as an singular vector of $\mathbf{H}_{K 2}$ and $\mathbf{b}_{K K}$ is taken as an singular vector of $\mathbf{H}_{K K}$.

\section{B. Zero-forcing and decoding}

The received signal contains three terms, the first term is the desired signal ,second term is the interference signal and the last part signifies the noise. We consider the conventional zero-forcing matrix $U_{i}$ to nullify the second term to zero. The zero-forcing matrix $U_{i}$ is chosen such a way that the desired column vectors are orthogonal to the vector space spanned by each other and to the interference space spanned at the i-th receiver.

Hence the zero-forcing filter at the first receiver $U_{1}$ has to be selected such a way that the vectors spanning desired signals $\left[\begin{array}{llll}x_{11} & x_{12} & \ldots & x_{1 K}\end{array}\right]$ are independent of each other and also orthogonal to the interference vectors. Hence the zeroforcing filter at first receiver is

$$
\mathbf{U}_{1}=\left[\begin{array}{llllllll}
\mathbf{H}_{11} \mathbf{b}_{11} & \mathbf{H}_{12} \mathbf{b}_{12} & \ldots & \mathbf{H}_{1 K} \mathbf{b}_{1 K} & \mathbf{H}_{11} \mathbf{b}_{21} & \ldots \mathbf{H}_{11} \mathbf{b}_{K 1}
\end{array}\right]
$$

For i-th receiver,the zero-forcing filter would be

$$
\mathbf{U}_{i}=\left[\begin{array}{lllllll}
\mathbf{H}_{i 1} \mathbf{b}_{i 1} & \mathbf{H}_{i 2} \mathbf{b}_{i 2} & \ldots & \mathbf{H}_{i K} \mathbf{b}_{i K} & \mathbf{H}_{i 1} \mathbf{b}_{j 1} & \ldots & \mathbf{H}_{i 1} \mathbf{b}_{K 1}
\end{array}\right]
$$

where $i, j=1, \ldots, K$ and $i \neq j$

To bringout the IA, we apply the receive filter $U_{i}$ to (). The outcome of this operation is given by

$$
\begin{gathered}
\hat{\mathbf{x}}_{i}=\mathbf{U}_{i}^{H} \mathbf{y}_{i} \\
\hat{\mathbf{x}}_{i}=\mathbf{U}_{i}^{H} \sum_{j=1}^{K} \mathbf{H}_{i j} \mathbf{b}_{i j} \mathbf{x}_{i j}+\underbrace{\mathbf{U}_{i}^{H} \sum_{j=1}^{K} \sum_{\substack{k=1 \\
k \neq i}}^{K} \mathbf{H}_{i j} \mathbf{b}_{k j} \mathbf{x}_{k j}}_{\text {Interference term }=0}+\mathbf{U}_{i}^{H} \mathbf{n}_{i}
\end{gathered}
$$

\section{Achievability of Degree of Freedom}

Since each receiver achieves the DoF of $\frac{K A}{2 K-1}$, the overall DoF of the proposed $K$-user $\mathrm{X}$ network achieved is $\frac{K^{2} A}{2 K-1}$. We consider the $K$-user MIMO X network, where the theoretical bound on the DoF region is defined as

$$
\frac{K^{2} A}{2 K-1 / A} \leq D o F \leq \frac{K^{2} A}{2 K-1}
$$

DoF of our proposed scheme falls in this region. The above mentioned settings sets Sum Rate of the proposed scheme as

$$
R_{\text {sum }}=\sum_{k} \frac{K}{2 K-1} E\left[\log \left(1+\left|h_{k k}\right|^{2}\right]\right.
$$




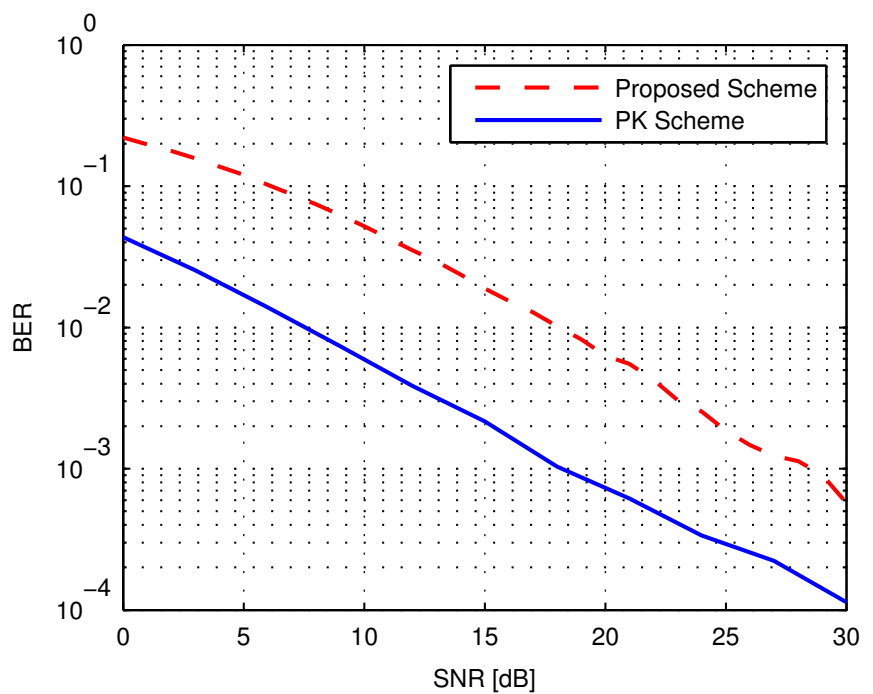

Fig. 2. BER vs SNR curve for proposed and PK scheme

\section{NUMERICAL RESULTS}

In this Section, the bit error rate (BER) of the proposed IA scheme is evaluated by means of Monte Carlo simulations. Assuming an average transmitted power from each antenna equal to $P_{\text {ave }}$, the signal-to-noise ratio is defined as $\mathrm{SNR}=$ $P_{\text {ave }} / \sigma_{n}^{2}$. As in [11], the coefficients of MIMO channels are assumed to remain constant for the entire duration of payload data transmission. Channel coefficients are independent and Rayleigh distributed with unit average power. Linear zeroforcing is applied at receivers side for detection [10].

We first consider the case where transmitted symbols $x_{i j}$ are chosen from a quadrature phase-shift keying (QPSK) constellation. In Figure 2 the BER performance of the proposed $K$-user X network IA scheme is compared to that of the $K$ user X network IA scheme discussed by Park and Ko (termed as PK Scheme). For a fair comparison we choose $K=3$. From the Figure it is possible to observe that for a fixed signal-to-noise ratio (SNR) our proposed scheme for a three-user $X$ network has a BER that is worse than that of PK Scheme. However, in the proposed scheme, each user achieves a DoF of $K A /(2 K-1)$ with $(2 K-1)$ antennas,whereas a similar DoF is achieved with $2 K$ antennas in PK scheme. It should be noted that DoF is also called as capacity pre-log, hence our proposed scheme achieves required capacity with fewer hardware.

In Figure 3 the BER performance of the IA of the twouser $\mathrm{X}$ network is compared to that of the proposed scheme for the $K$-user $\mathrm{X}$ network, where $K=3$. From the Figure it is possible to observe that for a fixed SNR the proposed threeuser TDMA scheme has a BER that is slightly worse than that of the conventional two-user $\mathrm{X}$ network. This is a predictable outcome as the increase in $K$ increases the error induced to the system. Though IA aligns interference at the receiver, the number of interfering users are increased with $K$, which would possibly increase error.

Figure 4 reports the capacity versus SNR for PK scheme and our proposed scheme. The curve is realized using equation (1). From the figure it is clear that PK scheme performs better

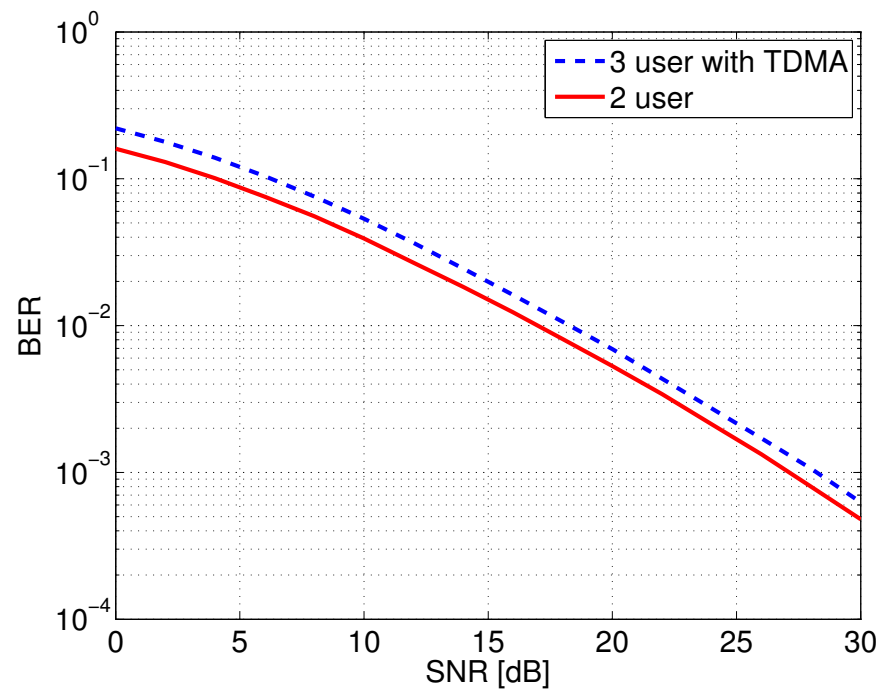

Fig. 3. BER vs SNR curve of $K=2$ and proposed scheme with $K=3$.



Fig. 4. Capacity vs SNR curve of $K=3$ for proposed scheme and PK scheme.

compared to our proposed scheme, but PK scheme has a restriction to use 6 antennas for this toy example with $K=3$, whereas though our proposed scheme shows lesser capacity, it complies with the scheme suggested by [4]. However another reason for higher capacity in PK scheme is the higher number of antennas used. As number of antenna (A) is a multiplier to the DoF $\frac{M N A}{M+N-1}$, an increase in A would linearly increase the capacity.

\section{CONCLUSION}

In this paper, an interference alignment scheme has been introduced for a $K$-user MIMO X channel. For the proposed scheme the alignment condition is achieved by means of a TDMA approach where shaping beamforming vectors at each transmitting node are obtained by using the knowledge associated with SVD of channel matrices between nodes. The proposed scheme allows to achieve degree of freedom of $\frac{K A}{2 K-1}$ per user by using $2 K-1$ antennas at each node. Hence sum DoF of the system is lesser comparing the PK scheme, but achieves the required DoF per time slot. As the sum DoF is lesser, the advantage of using IA would fade away at higher ' $\mathbf{K}$ '. The bit error rate performance of the 
proposed scheme is compared to that of the scheme proposed by Park and Ko for the three-user X channel. Simulation results are reported to show the BER performance for proposed scheme with $K=3$ and already existing scheme with $K=2$, with QPSK modulations in case of transmission over flatfading Rayleigh channels. Though the BER performance of proposed scheme is slightly worse compared to the two-user $\mathrm{X}$ network IA, our scheme lifts off the practical limitation of having Perfect IA in $K$-user MIMO $\mathrm{X}$ channel. The extension of the proposed interference alignment approach by retaining the sum DoF is still an open problem.

\section{REFERENCES}

[1] S. A. Jafar and S. Shamai, "Degrees of freedom region of the MIMO X channel," IEEE Trans. Inf. Theory, vol. 54, no. 1, pp. 151-170, Jan. 2008.

[2] V. R. Cadambe and S. A. Jafar, "Interference alignment and degrees of freedom of the K-user interference channel," IEEE Trans. Inf. Theory, vol. 54 , no. 8 , pp. $3425-3441$, Aug. 2008

[3] M. A. Maddah-Ali, A. S. Motahari, and A. K. Khandani, "Communication over MIMO X channels: interference alignment, decomposition, and performance analysis," IEEE Trans. Inf. Theory, vol. 54, no. 8, pp. 3457-3470, Aug. 2008.

[4] V. R. Cadambe and S. A. Jafar, "Interference alignment and the degrees of freedom of wireless X networks," IEEE Trans. Inf. Theory, vol. 55, no. 9 , pp. 3893-3908, Sep. 2009.
[5] C. Rose, S. Ulukus, and R. D. Yates, "Wireless systems and interference avoidance," IEEE Trans. Wireless Commun., vol. 1, no. 3, pp. 34573470 , July 2002.

[6] J. G. Andrews, W. Choi, and R. W. Heath Jr., " Overcoming interference in spatial multiplexing MIMO cellular networks," IEEE Wireless Commun., vol. 14, no. 6, pp. 95-104, Dec. 2007.

[7] S. A. Jafar, "Interference alignment: a new look at signal dimensions in a communication network," Foundations and Trends in Communication and Information Theory, vol. 7, no. 1, pp. 1-136, 2011.

[8] S. W. Peters, and R. W. Heath, "Interference alignment via alternating minimization," in Proc. Intern. Conf. Acoust., Speech, Signal Process., pp. 2445-2448, Apr. 2009.

[9] R. K. Mungara, G. George, and A. Lozano, "Overhead and spectral efficiency of pilot-assisted interference alignment in time-selective fading channels," IEEE Trans. Wireless Commun., vol. 13, no. 9, pp. 4884-4895, Sept. 2014.

[10] L. Bai and J. Choi, Low complexity MIMO detection. Springer, 2012.

[11] A. Mahmoud, M. El-Khamy, and K. Elsayed, "Interference alignment performance on MIMO X channels with imperfect channel knowledge," in Proc. Intern. Workshop Signal Proc. Adv. Wireless Commun., pp. 239-243, 2012.

[12] S.-H. Park and Y.-C. Ko, "K-user MIMO X network system with perfect interference alignment," in Proc. Intern. Conf. Commun., pp. 1-5, June 2011. 